\title{
The Role of Disjoining Pressure and Thermal Activation in the Invasion of Droplets into Nanopores
}

\author{
Chao Fang, ${ }^{\dagger}$ Qinjun Kang, ${ }^{\ddagger}$ and Rui Qiao, ${ }^{* \dagger}$ \\ ${ }^{\dagger}$ Department of Mechanical Engineering, Virginia Tech, Blacksburg, Virginia 24061, United States \\ ${ }^{\ddagger}$ Earth and Environmental Sciences Division, Los Alamos National Laboratory, Los Alamos, New Mexico 87545, United States
}

\section{Supporting Information}

ABSTRACT: Multiphase transport at a nanoscale level plays a key role in applications including drying of nanoporous materials and gas/oil recovery from low permeability rocks. A frequently encountered scenario in multiphase transport is the presence of droplets near nanopores. Whether droplets invade the nanopores or become trapped at their entrance greatly affects the operation of engineered systems. Here we analyze the free energy profile of nanometer-sized droplets entering the nanopore and how the profile is affected by the pressure difference and the size of the droplet and the nanopore. We show that, for nanopores whose surface is fully wetted by water but not the droplet, a droplet larger than the pore diameter must overcome a higher free energy barrier than that predicted by classical theories due to the large disjoining pressure. For smaller nanodroplets, the threshold pressure for their invasion into a given nanopore can be lowered by thermal activation. When a droplet is slightly narrower than a pore, and thus is often assumed to enter the pore freely, a large energy barrier for droplet entry can actually exist. The droplet cannot easily enter the pore even with hydrodynamic drag by moving fluids. Entering the pore through Brownian motion is possible, and the mean entry time depends sensitively on the pore size and can reach seconds or even longer. These findings provide molecular insights on the invasion of droplets into nanopores and lay foundations for large-scale modeling of multiphase nanofluidic transport.

\section{INTRODUCTION}

Multiphase transport at the nanoscale is widely encountered in engineering practice, e.g., in the drying of natural and engineering nanoporous materials, ${ }^{1,2}$ gas and oil recovery, ${ }^{3,4}$ and oil-water separation., 5 In such transport, the solid boundaries are often preferentially wetted by one fluid phase and another fluid phase is dispersed as bubbles or droplets. A frequently encountered scenario is the presence of an immiscible phase as a droplet/bubble near the entrance of a nanopore. Understanding how the droplet or bubble enters the nanopore is crucial because whether it can translocate the nanopore or become trapped at the entrance can greatly affect the operation and performance of practical systems. Knowledge of such transport is also necessary input for simulations such as the pore-scale modeling of multiphase flows in porous media.

Prior studies of the transport of droplets through narrow pores showed that the wetting property of the droplet phase on the pore surface, the size and shape of the droplet and pore, and the external pressure difference play important roles in determining the droplet transport behavior. ${ }^{7-13}$ For example, in the absence of a pressure difference between the up- and downstream of the pore, the free energy landscape for droplet transport has been investigated theoretically. If the droplet size is below a threshold value, which is comparable to the size of the pore, a nonwetting droplet can enter the pore spontaneously. This prediction has been verified in the adsorption of transition liquid metal droplets into carbon nanotubes, where various droplet transport regimes were identified. ${ }^{8,9}$ More droplet transport regimes were also identified when the spreading of droplets on the exposed solid surface outside the pore was taken into account. ${ }^{10}$ If the droplet size is considerably larger than the width of a pore, an external pressure difference is required to drive the droplet into the pore. The threshold invasion pressure can be estimated using the Laplace pressure difference between the front and rear sides of the droplet as it enters the pore. ${ }^{13}$ When strong flow is involved during the invasion of a droplet into a pore, the ensuing change of the curvature at the droplet's leading edge has also been shown to affect the droplet transport greatly. ${ }^{11}$

While prior works advanced the basic understanding of droplet transport into pores, new issues arise when the pores reach nanoscale dimensions. First, the application of the insights from prior studies involves uncertainties. When a nonwetting droplet enters a nanopore, a molecularly thin film can form between the droplet and the surface of the nanopore and a host of interfacial forces can emerge. To what extent

Received: January 10, 2019

Revised: February 20, 2019

Published: February 25, 2019 
these forces affect the free energy landscape of droplet transport into the nanopore is not clear a priori. For example, while droplets smaller than the nanopore size were expected to enter the nanopore freely in most previous studies, it is not clear if this is universally true for nanopores with various surface properties. Second, when an external pressure difference exists between the up- and downstream of the nanopore, much of the prior work focused on identifying the threshold pressure difference under which a droplet can deterministically enter the nanopore. However, when the size of the droplets and pores reaches nanoscale, where thermal fluctuations are significant, a droplet can potentially enter and translocate the nanopore by overcoming the energy barrier through thermal activation. Therefore, classifying the free energy landscape for droplet transport through nanopores in the presence of a pressure difference, which has received limited attention in prior works, becomes important.

Here, we are interested in how a droplet invades a nanopore whose surface is fully wetted by water. Our interest in this system is motivated by its relevance to gas/oil extraction from shales, where much of the gas/oil exists in pores with nanoscale dimensions and the surfaces of many pores are strongly hydrophilic. ${ }^{14-16}$ Much of the existing work on gas/oil transport in shales focuses on single phase transport. However, multiphase transport does occur during gas/oil recovery because water is found ubiquitously in shale formation and they can greatly affect the transport of gas/oil. ${ }^{17}$ While studying multiphase transport in nanopores experimentally is difficult, this problem is amenable to molecular dynamics (MD) simulations. In MD simulations, the nanopore and all fluids are resolved at the molecular level and the transport can be modeled with much less restrictive assumptions compared to continuum models. Indeed, MD simulations have emerged as a powerful tool for understanding nanoscale multiphase transport in recent years. ${ }^{4,18}$

In this study, we examine the free energy profile of a droplet as it enters the nanopore and how this profile is affected by the applied pressure difference and the size of the droplet and nanopore. We show that, for nanopores whose surface is completely wetted by water but not by the droplet, a droplet larger than the pore size must overcome a higher free energy cost than that predicted by classical theories due to large disjoining pressure. When the droplet is smaller than the pore size, and thus the droplet is usually expected to enter the pore freely, a large entry barrier for droplet entrance can nevertheless exist. The insight gained here helps aid the understanding of the nonlinear multiphase transport phenomena in nanoporous media and inform the development of better pore-scale models.

\section{MODELS AND METHODS}

We use continuum and molecular dynamics (MD) models to determine the free energy profile for the invasion of droplets into nanopores. In the continuum model, we consider twodimensional (2D) droplets invading a slit pore and threedimensional (3D) spherical droplets invading a cylinder pore. The $2 \mathrm{D}$ cases are studied to facilitate the assessment of the accuracy of continuum models by MD models because studying large droplets in 3D using $\mathrm{MD}$ simulations is much more expensive than in $2 \mathrm{D}$. As we shall see, the results in $2 \mathrm{D}$ and $3 \mathrm{D}$ spaces are qualitatively similar.

2.1. Continuum Model. We first examine the invasion of a droplet into a long pore in 2D space. A droplet with an initial diameter $D_{0}$ is immersed in water and positioned at the entrance of a pore with a width $W$. Figure 1a shows the sketch
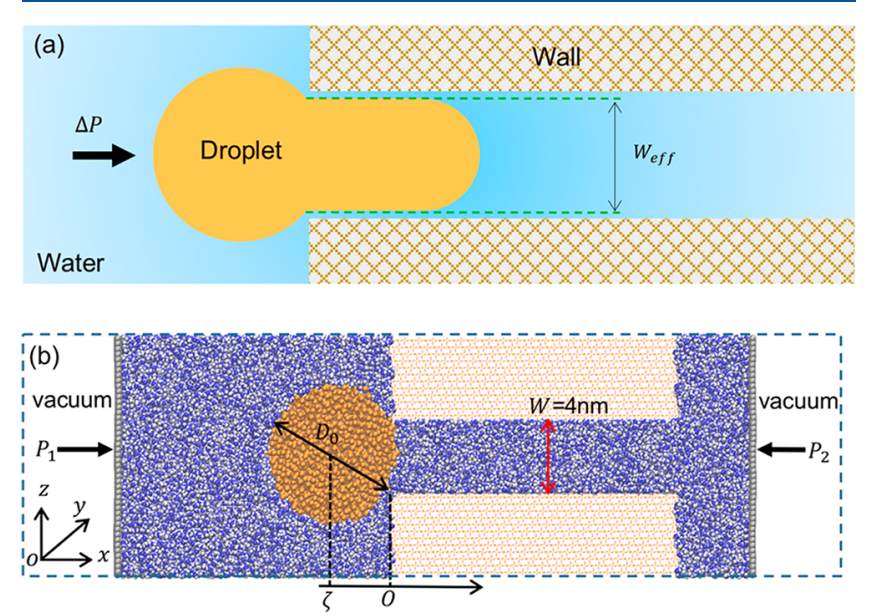

Figure 1. Continuum and molecular models of droplet invasion into a slit pore. (a) A schematic of the model for the invasion of a droplet into a pore. The dashed lines denote the boundary of the thin liquid film between the droplet and water after invasion. (b) A snapshot of a representative MD model. An un-deformed droplet (diameter: $\left.D_{0}\right)$ is initially positioned at the entrance of a slit pore (nominal width: 4 $\mathrm{nm}$ ). The pressure up- and downstream from the pore is set to $P_{1}$ and $P_{2}$ by the two pistons. The system is periodic in all three directions, but two large vacuum spaces are placed outside of the two pistons. Water molecules are shown as blue (oxygen) and white (hydrogen) spheres. The droplet (piston) atoms are shown as orange (gray) spheres. The pore walls made of quartz are shown as lines. The dashed box denotes the simulation box.

of such a droplet entering the pore. A pressure difference $\Delta P$ is imposed between the upstream reservoir and downstream pore. We focus on the situation where the pore wall is completely wetted by water and the droplet diameter is larger than the pore width. Hence, as the droplet enters the pore, a thin residual water film remains on the pore wall, which reduces the pore to an effective width $W_{\text {eff. }}$ We note that such thin water films, akin to the precursor film in front of a droplet spreading on a hydrophilic surface, ${ }^{19}$ are observed both in prior studies of bubbles/droplets entering pores with strongly hydrophilic walls (e.g., mica) $)^{20-22}$ and in our MD simulations (see below).

Following the standard approach,,$^{4,8-10,12,23}$ we determine the free energy profile as the droplet moves into the pore by considering only the surface energy and pressure work. For an infinitesimal displacement of the droplet into the pore, the associated free energy variation $\mathrm{d} G$ is thus

$$
\mathrm{d} G=\gamma \mathrm{d} A-\Delta P \mathrm{~d} V_{\text {in }}
$$

where $\gamma$ is the water-droplet interfacial tension and $\mathrm{d} A$ and $\mathrm{d} V_{\text {in }}$ are the changes of the area of water-droplet interface and the volume of the droplet inside the pore with the displacement of the droplet into the pore, respectively. To obtain the free energy profile, three widely used approximations are made: the droplet is pinned at the pore mouth before fully entering the pore; with the exception of the interface between the droplet and the residual water film, other water-droplet interfaces are circular; the droplet is incompressible. $^{8-10,12}$ The details for evaluating the free energy profile are provided in Figure $S 1$ and the associated text in the 

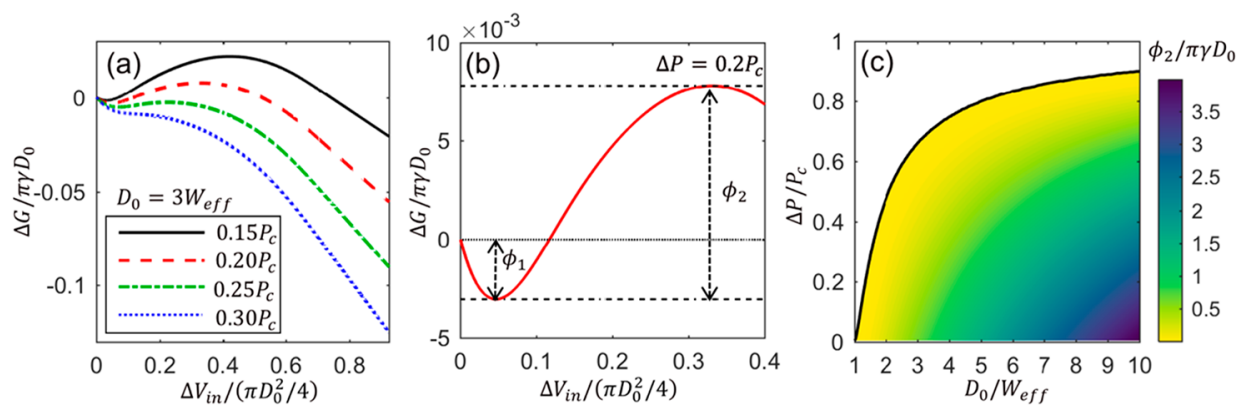

Figure 2. Continuum model of droplet invasion into a slit pore. (a) The free energy profiles of an invading droplet as a function of $\Delta V_{\text {in }}$ under three pressure differences along the pore $\left(\Delta V_{\text {in }}=V_{\text {in }}-V_{\text {in, },}\right.$, where $V_{\text {in }}$ and $V_{\text {in }, 0}$ are the volume of the droplet inside the pore at any instant during invasion and at the instant when the droplet first touches wall edges, respectively). The diameter of the un-deformed droplet, $D_{0}$, is $3 W_{\text {eff }}$ (b) $\mathrm{A}$ zoomed-in view of the free energy profile for $\Delta P=0.2 P_{c}$. (c) Dependence of the entry barrier height $\phi_{2}$ on the droplet size and applied pressure. The solid line corresponds to $\phi_{2}=0$.

Supporting Information. After studying the droplet invasion in $2 \mathrm{D}$, the calculations are repeated in $3 \mathrm{D}$ space.

2.2. MD Models and Methods. Figure $1 \mathrm{~b}$ shows a snapshot of a representative MD system. The system consists of two water reservoirs, a slit channel bounded by two solid walls, a droplet immiscible with water, and two rigid pistons. The pressure in the up- and downstream reservoirs is maintained by applying appropriate forces on the two pistons bounding them. The system is periodic in all three directions, but two large vacuum spaces are placed outside of the pistons to effectively remove the periodicity in the $x$-direction. The droplets are cylindrical in shape because they span the periodic MD box in the $y$-direction. Droplets with diameters of 1.86 , $2.26,2.60$, and $6.00 \mathrm{~nm}$ have been studied. The full dimensions of the pore and the vacuum space are varied for different droplets and are summarized in Table S1 in the Supporting Information. With the droplet and simulation box sizes listed in Table S1, finite-size effects on the free energy profile for droplet invasion should be minor in our simulations (see Figure S2 in the Supporting Information and related discussion). The pressure applied on the right piston is fixed at 1 bar, while that on the left piston is varied in different simulations to generate different pressure differences up- and downstream from the slit pore.

Water is described using the SPC/E model. ${ }^{24}$ The droplets are made of Lennard-Jones (LJ) particles with $\mathrm{LJ}$ parameters the same as those for methane molecules ${ }^{25}$ except that the $\epsilon$ value for droplet-droplet interactions is set to $4.0 \mathrm{~kJ} / \mathrm{mol}$ in our simulation so that the droplet behaves like liquids. The LJ parameters for the interactions between the droplet atoms and all other types of atoms in the system are obtained using the Lorentz-Berthelot combination rule. The channel wall is made of $\alpha$-quartz cleaved from the (101) direction, with a silanol group surface density of 5.92 per $\mathrm{nm}^{2}$. The force field parameters including the Lennard-Jones potential and partial charges of quartz are taken from the CLAYFF force fields. ${ }^{26,27}$ The cleavage of the quartz surface follows recent work by other researchers. ${ }^{27}$ Following their convention, the zero plane of the quartz surface is defined as the location of the second outmost layer of the silicate atoms. The nominal channel width $W$ is the distance between the zero planes of the two walls (see Figure 1b). The quartz surface used here is strongly hydrophilic, as demonstrated by a separate simulation in which a water droplet initially placed above it ultimately spreads completely to form a thin film (see Figure S3 in the Supporting Information). To reduce computational cost, all of the silicate and oxygen atoms of the wall are fixed and their interaction with each other is excluded. The hydrogen atoms of surface silanol groups are allowed to vibrate by considering the bonds and angles of the silanol groups.

MD simulations are performed using the 5.1.4 version of the Gromacs package. ${ }^{28}$ The bonds and angles of water are constrained by the SETTLE algorithm. An NVT ensemble with the velocity-rescale thermostat and a time constant of 1 ps at $300 \mathrm{~K}$ is adopted. ${ }^{29}$ A global cutoff of $1.2 \mathrm{~nm}$ is used for computing the LJ potential, and the particle mesh Ewald (PME) method is used to calculate the electrostatic interactions. $^{30}$

The free energy profile for droplet invasion into the slit pore is computed using the umbrella sampling technique. ${ }^{31}$ The idea is to sample the free energy in different windows centered along the reaction coordinate of droplet invasion, which is taken as the center-of-mass position of the droplet. To generate initial configurations corresponding to droplets residing in different windows, we first apply a force to each atom of the droplet so that it moves at a speed of $\sim 1 \mathrm{~nm} / \mathrm{ns}$ from the left reservoir into the pore's interior. Configurations recorded during this nonequilibrium run are then used as the initial configurations of the umbrella sampling runs. A window size of 0.15 and $0.20 \mathrm{~nm}$ is used for the small and large droplets, respectively. Next, equilibrium umbrella sampling runs are performed so that the position of the droplet in the $x$ direction in each window is sampled for $20-30$ ns. In these simulations, a force is applied on each atom of the droplet to constraint the droplet's center-of-mass around the center of each sampling window. This force is given by $F_{\text {com }}=K\left(x_{\text {com }}-\right.$ $\zeta)^{2}$, where $x_{\text {com }}$ and $\zeta$ are the droplet's center-of-mass and the center of the sampling window in the $x$-direction, respectively. $K$ is a spring constant taken as $1000 \mathrm{~kJ} / \mathrm{mol} \cdot \mathrm{nm}^{2}$. From the histograms of the center-of-mass of the droplet in each sampling window (see Figure S4 in the Supporting Information), the potential of mean force (PMF) of the droplet is computed using the weighted histogram analysis method and the error bar is estimated using the bootstrap analysis with 100 bootstraps. ${ }^{32}$

For comparing the free energy profile of droplet invasion obtained from continuum and MD models, the interfacial tension between the water and droplet phase is needed. This interfacial tension is calculated from a separate simulation. In this simulation, a slab of LJ particles with $\mathrm{LJ}$ parameters identical to the droplet is sandwiched between two slabs of water and the periodic MD box has a cross-sectional area of 4 
$\times 4 \mathrm{~nm}^{2}$ and a thickness of $8 \mathrm{~nm}$ in the direction normal to the LJ particle-water interface (the $x$-direction). An equilibrium NVT simulation is performed at $300 \mathrm{~K}$ for $8 \mathrm{~ns}$. The interfacial tension is e valuated using $\gamma=1 / 2 \int_{0}^{L_{x}}\left[P_{x x}-0.5\left(P_{y y}+P_{z z}\right)\right] \mathrm{d} x,^{33}$ where $L_{x}$ is the box length in the $x$-direction and $P_{x x}, P_{y y}$, and $P_{z z}$ are the three diagonal components of the pressure tensor along the $x$-, $y$-, and $z$-directions. The interfacial tension is determined as $94.84 \pm 4.94 \mathrm{mN} / \mathrm{m}$.

\section{RESULTS AND DISCUSSION}

3.1. Continuum Models. Figure 2a shows the evolution of the free energy $\Delta G$ as a function of the droplet volume $\Delta V_{\text {in }}$ invading into the pore for the case of $D_{0}=3 W_{\text {eff }}$ at several pressure differences $\Delta P$. For generality, $\Delta G, \Delta V_{\text {in }}$ and $\Delta P$ are normalized using $G_{0}=\pi \gamma D_{0}, V_{0}=\pi D_{0}^{2} / 4$, and $P_{c}=2 \gamma / W_{\text {eff }}$ respectively. For large $\Delta P$ (e.g., $\Delta P=0.3 P_{\mathrm{c}}$ ), the free energy decreases monotonically as $\Delta V_{\text {in }}$ increases, and the droplet can invade the pore spontaneously. For low $\Delta P$, the free energy profile exhibits two local extremums, which lead to an initial free energy valley (depth: $\phi_{1}$ ) and a subsequent energy barrier (height: $\phi_{2}$, see Figure $2 \mathrm{~b}$ ). Therefore, a droplet tends to be trapped near the pore mouth. Because whether the droplet can enter the pore is governed by the energy barrier height $\phi_{2}$, we extend the above calculations to different droplet sizes and pressure differences to compute a map of this barrier height. Figure $2 \mathrm{c}$ shows that, for a given droplet size and pore width, a spontaneous invasion pressure $\Delta P_{\mathrm{si}}$ exists: for $\Delta P \geq \Delta P_{\mathrm{si}}$, a droplet experiences no barrier as it invades the pore; for $\Delta P<$ $\Delta P_{\text {si }}$ a droplet must overcome a barrier to invade the pore, and this barrier increases as $\Delta P$ becomes smaller. As the size of the droplet relative to the pore increases (i.e., as $D_{0} / W_{\text {eff }}$ increases), $\Delta P_{\mathrm{si}}$ approaches the capillary pressure $P_{\mathrm{c}}$ and the barrier height for droplet invasion increases for a fixed $\Delta P$. In addition to $D_{0} / W_{\text {eff }}$ the barrier height tends to be higher for larger droplets and larger surface tension (note that $\phi_{2}$ is scaled by $G_{0}=\pi D_{0} \gamma$ in Figure $2 \mathrm{c}$ ).

The above analysis can be extended to three dimensions (see the Supporting Information), in which a spherical droplet invades a cylindrical pore, a situation often encountered in nanofluidic systems. To appreciate the absolute magnitude of the free energy landscape, the analysis is done under conditions typical of oil recovery operations; i.e., the temperature is $373 \mathrm{~K}$ and the interfacial tension $\gamma$ between droplet and water is $35 \mathrm{mN} / \mathrm{m}^{34}$ The key features of the free energy landscape for the droplet invasion here are similar to those revealed in Figure 2. For example, Figure 3a shows the map of the barrier height $\phi_{2}$ when a $6 \mathrm{~nm}$ diameter droplet enters pores with different sizes (note that $\phi_{2}$ is shown in units of thermal energy $k_{\mathrm{B}} T$, where $k_{\mathrm{B}}$ is the Boltzmann constant and $T$ is the temperature). As in the $2 \mathrm{D}$ case, a spontaneous invasion pressure $\Delta P_{\mathrm{si}}$ exists for each effective pore diameter $D_{\text {eff }}$ and a barrier must be overcome at lower pressure differences. Although $\phi_{2}$ can reach thousands of $k_{\mathrm{B}} T$, because of the droplet's small size, $\phi_{2}$ will not be prohibitively high if the pressure difference is not too much smaller than $\Delta P_{\mathrm{si}}$. For example, the red line in Figure $3 \mathrm{a}$ corresponds to a barrier height of $40 k_{\mathrm{B}} T$. Because the activation time is $\tau_{\mathrm{a}} \sim t_{0} \exp \left(G_{\mathrm{B}} /\right.$ $\left.k_{\mathrm{B}} T\right)\left(t_{0} \sim h / k_{\mathrm{B}} T, h\right.$ is the Planck's constant and $G_{\mathrm{B}}$ is the barrier height), a droplet under the action of thermal fluctuations can overcome this barrier to enter the pore after a waiting time of $\sim 8.3 \mathrm{~h}$ at the pore entrance. Hence, although
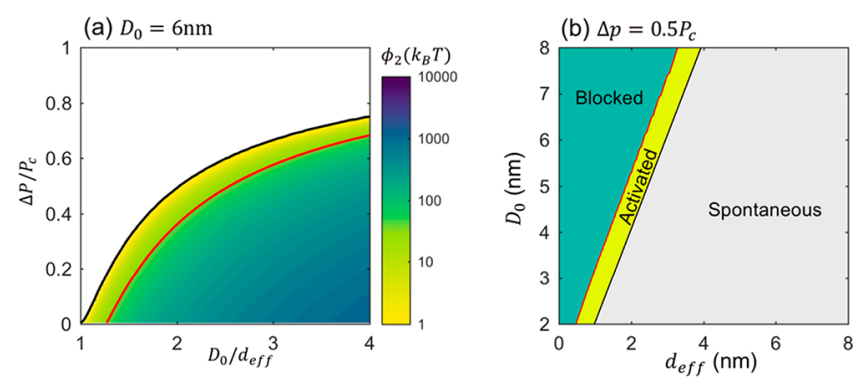

Figure 3. Invasion of a cylindrical pore by spherical droplets. (a) Dependence of the entry barrier height $\phi_{2}$ on the droplet size and pressure difference along the pore. The black and red lines correspond to $\phi_{2}=0$ and $\phi_{2}=40 k_{\mathrm{B}} T$, respectively. (b) A map for the droplet invasion mode as a function of the droplet and pore size. The pressure difference along the pore is fixed at $\Delta P=0.5 P_{\mathrm{c}}$.

the nanometer-sized droplet cannot enter the pore when $\Delta P<$ $\Delta P_{\mathrm{si}}$ in the deterministic view, it can nevertheless invade the pore in a stochastic view when $\Delta P$ is not too low compared to $\Delta P_{\text {si }}$; i.e., thermal activation lowers the threshold pressure for nanometer-sized droplets to invade nanopores. Equivalently, for a given pressure difference, thermal activation allows nanometer-sized droplets to invade pores smaller than that anticipated in the deterministic view. To show this, we computed the entry barrier height for a series of droplets and nanopores when the pressure difference is $50 \%$ of the capillary pressure. Without losing generality, we then assumed that a droplet can invade a pore by thermal activation if the entry barrier height is less than $40 k_{\mathrm{B}} T$ but is blocked otherwise. Figure $3 \mathrm{~b}$ shows the map of the droplet invasion mode as a function of droplet and nanopore size. We observe that, because of thermal activation, the droplets considered here can invade pores $\sim 0.5 \mathrm{~nm}$ (the width of the activated regime) narrower than that anticipated deterministically.

3.2. Molecular Models. Because the MD modeling of large spherical droplets is computationally expensive and droplet invasion in $2 \mathrm{D}$ and $3 \mathrm{D}$ space has similar features, we study the invasion of a slit pore by a nanodroplet that is periodic in the transverse direction (see Figure $1 \mathrm{~b}$ ), which is similar to the invasion of a $2 \mathrm{D}$ droplet into a slit pore studied in the previous section. Figure 4 shows the free energy profile of the droplet invasion under four pressure ratios. The free

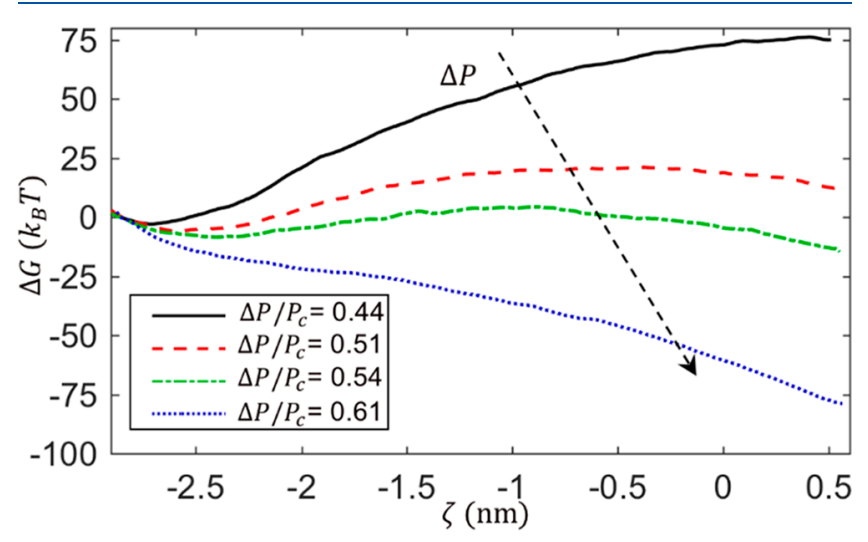

Figure 4. Molecular model of nanodroplet invasion into a slit pore. The free energy profiles of a $6 \mathrm{~nm}$ diameter droplet as a function of its center-of-mass position $(\zeta)$ at four different pressure differences from the molecular model. The upper bound of the error bar of the free energy at any point along the pore is $1.44 k_{\mathrm{B}} T$. 
energy is taken as zero at the position where the droplet just touches the wall edges. The pressure differences are again scaled using the capillary pressure of the pore $P_{\mathrm{c}}=2 \gamma / W_{\text {eff }}$ (the value of $W_{\text {eff }}$ is given below). The free energy profiles are qualitatively similar to these predicted by the continuum model. For instance, at $\Delta P=0.44 P_{\mathcal{c}}$ a significant entry barrier corresponding to a blockage of the droplet by the pore is observed. As the pressure difference rises to $0.61 P_{c}$, the free energy decreases monotonically as the droplet invades the pore, suggesting that the droplet invasion is spontaneous. For intermediate pressure differences, an initial trap and a subsequent barrier are observed, just as in Figure $2 \mathrm{~b}$.

To compare the MD predictions with those by continuum models, we first obtain the effective width of the slit pore. Figure 5a shows a snapshot of the droplet invading partially
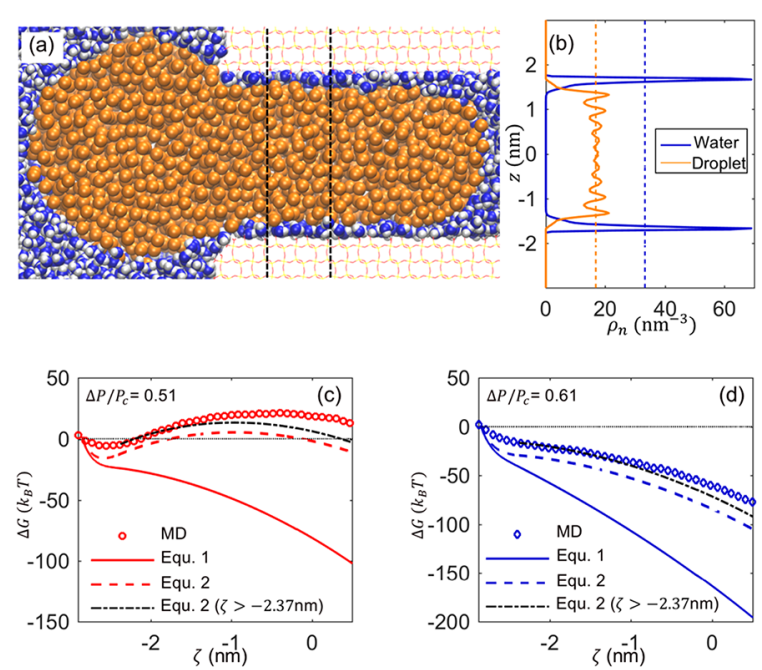

Figure 5. Comparing the MD predictions with continuum models. (a) A snapshot of the droplet invading partially into the slit pore in Figure $1 \mathrm{~b}$. The color coding is the same as that in Figure 1b. (b) The number density profiles of water and droplet across the slit pore in the region bounded by two dashed lines in panel a. The bulk water and droplet densities are shown as dashed lines. (c, d) The free energy profiles computed from continuum models and MD simulations at a pressure difference of $0.51 P_{\mathrm{c}}(\mathrm{c})$ and $0.61 P_{\mathrm{c}}(\mathrm{d})$. The upper bound of the error bar of the free energy from MD simulations at any point along the pore is $1.44 k_{\mathrm{B}} T$.

into the pore. Because of the strong hydrophilicity of the quartz wall, a thin water film is observed between the droplet and each pore wall. This effective width of the pore is evaluated from the number density profile of the water and droplet phase across the channel, which is calculated in the region between the two dashed lines in Figure 5a. As shown in Figure 5b, water accumulates near the solid wall and the density of the droplet phase is nearly constant in the middle part of the pore. Following the concept of Gibbs' dividing surface, the effective pore width $W_{\text {eff }}$ is found to be $2.93 \mathrm{~nm}$ by dividing the total mass of the droplet phase across the channel (computed from the density profile in Figure $5 b$ ) by the droplet phase's bulk density.

Parts $\mathrm{c}$ and $\mathrm{d}$ of Figure 5 show the continuum and MD free energy profiles of the droplet invading the nanopore at a pressure difference of $0.51 P_{\mathrm{c}}$ and $0.61 P_{\mathcal{C}}$, respectively. The MD predictions are systematically higher than the continuum predictions, which suggests that the continuum model tends to underestimate the threshold pressure for droplet invasion. We propose that the deviation of the continuum predictions from the $\mathrm{MD}$ predictions originates from the fact that the thin water films sandwiched between the droplet and the pore walls contribute to an additional energy term neglected in the continuum model. In the continuum model described above, the effect of water film is considered only through the surface energy of the water-droplet interfaces (see eq 1). Such an approach is acceptable if the water film is thick. Here, the water film is molecularly thin and intersurface interactions contribute greatly to the system's energy, which is lumped as the disjoining pressure effects in the literature. ${ }^{35-38}$ Therefore, as a droplet invades a pore, an extra term related to the disjoining pressure must be introduced into eq 1 to account for the energy required (gained) to form the thin water film

$$
\mathrm{d} G=\gamma \mathrm{d} A-\Delta P \mathrm{~d} V_{\text {in }}+E_{\mathrm{d}}(\delta) \mathrm{d} A^{\prime}
$$

where $E_{\mathrm{d}}(\delta)$ is the energy per unit area of a water film with a thickness of $\delta$ and an area of $A^{\prime}$. Assuming that the disjoining pressure is dominated by van der Waals forces, $E_{\mathrm{d}}(\delta)=A_{\mathrm{H}}$ / $12 \pi \delta^{2}$, where $A_{\mathrm{H}}$ is the Hamaker constant. The thickness of the water film behind the semicircle-shaped front of the droplet is taken as $0.23 \mathrm{~nm}$, which is determined using the water density profile shown in Figure $5 b$ and the concept of the Gibbs dividing surface. Using a typical value of the Hamaker constant for water on strong hydrophilic substrates $A_{\mathrm{H}} \sim 2.0 \times 10^{-20}$ $\mathrm{J},{ }^{36}$ the free energy profiles for droplet invasion are computed (see Figure $5 \mathrm{c}$ and $\mathrm{d}$ ). We observe that, when the disjoining pressure effects are considered, agreement between continuum and $\mathrm{MD}$ predictions is greatly improved. The remaining deviation is mostly caused by the fact that the energy variation during the initial phase of droplet invasion $(\zeta<-2.37 \mathrm{~nm}$, when the front portion of the droplet inside the pore has not yet formed a semicircle) is not captured well. Indeed, if eq 2 is used only in the region $\zeta>-2.37 \mathrm{~nm}$ and $\Delta G$ at $\zeta=-2.37 \mathrm{~nm}$ is set to the MD value, the agreement between continuum and $\mathrm{MD}$ predictions is very good (see Figure $5 \mathrm{c}$ and $\mathrm{d}$ ). The fact that the energy variation in the initial phase of droplet invasion is not captured well by the simple model used here is not surprising; e.g., the structure and energetics of the molecules in the water film near the sharp edge of pore entrance should differ from those in extended water films of same thickness, but such effects are not considered. We note that, apart from the disjoining pressure of the thin film, other physics including curvature effects and line tension can also be significant as the droplet dimension reaches nanometer scale. ${ }^{39}$ They may contribute to the discrepancy between eq 1 and MD results and can potentially explain why the corrected continuum model using eq 2 still cannot fully capture MD results.

The greatly improved agreement between the continuum and $\mathrm{MD}$ predictions shown above highlights the potential importance of disjoining pressure in the invasion of droplets into nanopores. For the disjoining pressure effects to be prominent, its contribution, which depends on the droplet and pore size, should be at least comparable to the combined pressure and surface tension effects considered in eq 1 . As shown in the Supporting Information, one can show that this amounts to requiring

$$
\alpha=\left|1-W_{\text {eff }} / 2 R_{0}-\Delta P / P_{\mathrm{c}}\right|^{-1} E_{\mathrm{d}}\left(\delta_{0}\right) / \gamma \gtrsim O(1)
$$

where $\delta_{0}$ is the thickness of the water film behind the front of the droplet inside the pore. When $\Delta P=\Delta P_{\text {si }}$, the prefactor I1 $-W_{\text {eff }} / 2 R_{0}-\Delta P / P_{\mathrm{c}} \mathrm{c}^{-1}$ approaches infinity. This is consistent with the idea that the effect of disjoining pressure on droplet 
invasion tends to be most significant at a $\Delta P$ value where a droplet can spontaneously invade a pore in the absence of such effects. For the system modeled here $E_{\mathrm{d}} / \gamma \sim 0.06$, the prefactors are 10 and 600 for $\Delta P / P_{c}=0.51$ and 0.61, separately. Therefore, $\alpha=0.6(36)$ when $\Delta P / P_{c}=0.51(0.61)$, which is consistent with the importance of disjoining pressure effects revealed in Figure 5c and d.

We next examine the invasion of droplets into pores wider than them. Figure 6 shows the free energy profiles for the

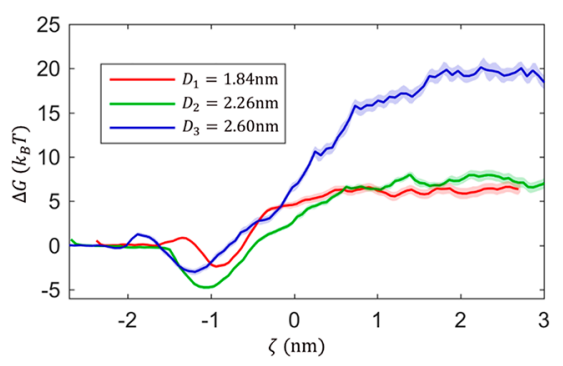

Figure 6. Invasion of nanodroplets into pores wider than them. Free energy profiles for the invasion of nanodroplets with diameters of $1.84,2.26$, and $2.60 \mathrm{~nm}$ into a slit pore with a nominal width of 4.0 $\mathrm{nm}$. The pressure difference between the up- and downstream of the pore is zero. The shallow shaded areas represent the errors of the free energy profile at each point.

invasion of droplets with diameters of $1.84,2.26$, and $2.60 \mathrm{~nm}$ into a pore with a nominal width of $4 \mathrm{~nm}$. No pressure difference is applied along the pore. Apart from a minor valley near the pore entrance, which is likely caused by the reduced hydrophilicity of the pore entrance's sharp edge, the free energy of the droplet inside the pore is $6-20 k_{\mathrm{B}} T$ above that in the bulk water. The latter suggests that, although it is often expected that a droplet can invade a pore wider than its diameter without any energy cost, for droplets invading pores only a few nanometers wider than them, an energy cost is involved. The existence of this cost and its increase with the droplet size are consistent with the disjoining pressure effects presented above. Because a circular droplet invading a pore wider than it barely changes shape and hence the waterdroplet interfacial area, no energy cost is expected. However, if the pore is only marginally wider than the droplet, the invasion of the droplet creates thin water films above and beneath it, which incurs disjoining pressure and related energy cost, as presented in eq 2 . Because the cost of creating these films increases as the films become thinner, the cost for a droplet invading the pore increases as the droplet size increases.

An interesting aspect of the invasion of droplets into wider pores is that the pressure drop between the up- and downstream of the pore no longer occurs mostly across the droplet, since the invading droplet no longer completely blocks the pore. Therefore, the invasion of a droplet into a pore relies on the hydrodynamic force exerted on it by the fluid flow and diffusion. Because the fluid velocity in a nanofluidic system is generally small $\left(\sim \mathrm{O}\left(10^{-7}-10^{-3} \mathrm{~m} / \mathrm{s}\right)\right)$, the hydrodynamic force is unlikely to be strong enough to overcome the thermodynamic force and drive the droplet into the pore. Here, we perform nonequilibrium simulations by imposing a pressure difference of $5 \mathrm{MPa}$ between the two reservoirs connected by the $4 \mathrm{~nm}$ wide pore (see Figure $1 \mathrm{~b}$ ). A $1.84 \mathrm{~nm}$ diameter droplet is initially placed in front of the pore entrance, and both the flux of water through the pore and the position of the droplet are monitored. The droplet remains trapped near the pore entrance during the $20 \mathrm{~ns}$ run (see Figure S6 in the Supporting Information). The mean water velocity through the pore is determined as $0.196 \mathrm{~m} / \mathrm{s}$ by linear regression of the ensemble average of the cumulative number of water molecules leaving the upstream reservoir (see the inset of Figure S6 in the Supporting Information). Using this mean velocity and the continuum prediction of the drag experienced by a $2 \mathrm{D}$ droplet confined in a slit channel, ${ }^{40}$ the upper bound of the hydrodynamic force exerted on the droplet is estimated to be $\sim 33 \mathrm{pN}$ (see the Supporting Information). The fact that this force is smaller than the maximal thermodynamic force repelling the droplet from the pore $(\sim 55 \mathrm{pN}$, determined from the free energy profile in Figure 6) is consistent with the observation that the droplet does not invade into the pore. In practical nanofluidic systems, the fluid velocity is generally less than $\sim 10^{-3} \mathrm{~m} / \mathrm{s}$, and thus, the hydrodynamic drag will be even weaker than that simulated here and thus the invasion driven by flow is more unlikely.

Although the droplets studied here are unlikely to invade the pore by fluid flow, invasion by thermal activation is possible. Using the free energy profiles shown in Figure 6 and assuming that the droplet invasion into a pore is a Brownian translocation process through a domain characterized by a free energy profile $\Delta G$, we can estimate the average time for a droplet near the pore entrance to enter the pore using ${ }^{41}$

$$
\begin{aligned}
\langle\tau\rangle= & \frac{1}{D} \int_{\xi_{\mathrm{s}}}^{\xi_{\mathrm{e}}} \exp \left(\Delta G(\xi) / k_{\mathrm{B}} T\right) \mathrm{d} \xi \\
& \int_{\xi_{\mathrm{s}}}^{\xi} \exp \left(-\Delta G(x) / k_{\mathrm{B}} T\right) \mathrm{d} x
\end{aligned}
$$

where $D$ is the droplet's diffusion coefficient. $\xi_{\mathrm{s}}$ is the left domain boundary, where $\Delta G$ first reaches zero if the droplet approaches from the pore interior to the pore outside. $\xi_{\mathrm{e}}$ is the right domain boundary, where $\Delta G$ first reaches $\phi_{2}$ if the droplet approaches from the pore interior from outside of the pore. The diffusion coefficients of the three droplets inside the slit pore are evaluated using separate simulations as $0.51 \times$ $10^{-9}, 0.45 \times 10^{-9}$, and $0.37 \times 10^{-9} \mathrm{~m}^{2} / \mathrm{s}$ from the smallest to the largest droplet (see Figure S8 in the Supporting Information). The average entry times are estimated to be $9.81 \times 10^{-7}, 1.17 \times 10^{-4}$, and $4.91 \mathrm{~s}$ for the droplets with a diameter of $1.84,2.23$, and $2.60 \mathrm{~nm}$, respectively.

The invasion of spherical droplets into cylindrical pores can be understood using the free energy profiles computed for cylindrical droplets invading slit pores. Specifically, assuming that the disjoining pressure in a liquid film depends solely on its local thickness, the free energy profile for the invasion of a spherical droplet into a cylindrical pore $\Delta G_{3 \mathrm{D}}(\zeta)$ is related to that for the invasion of a cylindrical droplet with the same diameter and a length of $L_{y}$ into a slit pore with width equal to the circular pore diameter, $\Delta G(\zeta)$, by $\Delta G_{3 \mathrm{D}}(\zeta)=\pi \Delta G(\zeta) D_{0} /$ $2 L_{y}$, where $D_{0}$ is the droplet diameter (see the Supporting Information). Using this relation, the free energy profiles in Figure 6, and eq 4, the average entry time for spherical droplets with diameters of $1.84,2.26$, and $2.60 \mathrm{~nm}$ to enter a $4 \mathrm{~nm}$ diameter cylindrical pore can be estimated as $1.80 \times 10^{-7}, 3.84$ $\times 10^{-5}$, and $1.15 \times 10^{1} \mathrm{~s}$, respectively. These results and those for the $2 \mathrm{D}$ droplet show that, for droplets entering pores slightly wider than them through Brownian diffusion, the entry time can increase sharply as the droplet size approaches the pore size and may reach minutes or even longer. 


\section{CONCLUSION}

In summary, we study the invasion of droplets into nanopores using molecular and continuum simulations. Depending on the applied pressure and the relative size of the pore and the droplet, three regimes can be found: spontaneous invasion, invasion by thermal activation, and blocking at the pore entrance. The most interesting scenario occurs when the droplets have nanometer sizes and the pore width is comparable to the droplet diameter. When the droplet size is larger than the pore size, thermal activation can modestly expand the parameter space in which a droplet can invade the pore compared to deterministic expectations. However, the disjoining pressure associated with the thin water film on the pore wall generally hinders the droplet invasion by driving up the energy barrier. In particular, when the droplet diameter is smaller than the pore size, disjoining pressure can lead to notable energy barriers for droplet invasion. These barriers are unlikely to be overcome by the hydrodynamic drag on the droplet but may be crossed through the Brownian motion of the droplet. In that case, the droplet entry time can increase dramatically as the droplet diameter approaches the pore size.

Our study highlights that, as droplets and the liquid films formed between the invading droplets and pore walls approach nanoscale dimensions, physics insignificant under other conditions, e.g., thermal activation and droplet blocking by wide pores due to the disjoining pressure, can become important. Understanding these nonlinear phenomena helps understand the non-Darcy multiphase flow behavior in nanoporous media and also helps improve the modeling of multiphase transport in unconventional porous media. For example, understanding the different invasion regimes and the spontaneous invasion pressure difference helps improve the pore-scale models to achieve more accurate prediction of droplet transport in shale reservoirs.

\section{ASSOCIATED CONTENT}

\section{S Supporting Information}

The Supporting Information is available free of charge on the ACS Publications website at DOI: 10.1021/acs.jpcc.9b00287.

Calculation of the free energy profile for droplet invasion by continuum theory, MD system parameters, assessment of finite-size effects, wetting properties of the quartz walls, representative umbrella sampling data, assessment of disjoining pressure effects on droplet invasion, nonequilibrium simulation of droplet invasion into a wide pore, the diffusion coefficients of small droplets in slit pores, and the free energy profiles of initially spherical droplet invading cylindrical pores (PDF)

\section{AUTHOR INFORMATION}

\section{Corresponding Author}

*E-mail: ruiqiao@vt.edu.

\section{ORCID $\odot$}

Rui Qiao: 0000-0001-5219-5530

\section{Notes}

The authors declare no competing financial interest.

\section{ACKNOWLEDGMENTS}

We thank the ARC at Virginia Tech for generous allocations of computer time. R.Q. and C.F. acknowledge the support by US
DOE through grant DE-NE0008806. Q.K. acknowledges the support from the Laboratory Directed Research and Development (LDRD) Program of Los Alamos National Laboratory.

\section{REFERENCES}

(1) Tas, N. R.; Mela, P.; Kramer, T.; Berenschot, J. W.; van den Berg, A. Capillarity induced negative pressure of water plugs in nanochannels. Nano Lett. 2003, 3 (11), 1537-1540.

(2) Duan, C.; Karnik, R.; Lu, M.-C.; Majumdar, A. Evaporationinduced cavitation in nanofluidic channels. Proc. Natl. Acad. Sci. U. S. A. 2012, 109 (10), 3688-3693.

(3) Wang, L.; Tian, Y.; Yu, X.; Wang, C.; Yao, B.; Wang, S.; Winterfeld, P. H.; Wang, X.; Yang, Z.; Wang, Y. Advances in improved/enhanced oil recovery technologies for tight and shale reservoirs. Fuel 2017, 210, 425-445.

(4) Lee, T.; Bocquet, L.; Coasne, B. Activated desorption at heterogeneous interfaces and long-time kinetics of hydrocarbon recovery from nanoporous media. Nat. Commun. 2016, 7, 11890.

(5) Zheng, X.; Guo, Z.; Tian, D.; Zhang, X.; Li, W.; Jiang, L. Underwater self-cleaning scaly fabric membrane for oily water separation. ACS Appl. Mater. Interfaces 2015, 7 (7), 4336-4343.

(6) Gao, C.; Sun, Z.; Li, K.; Chen, Y.; Cao, Y.; Zhang, S.; Feng, L. Integrated oil separation and water purification by a double-layer $\mathrm{TiO}$ 2-based mesh. Energy Environ. Sci. 2013, 6 (4), 1147-1151.

(7) Marmur, A. Penetration of a small drop into a capillary. J. Colloid Interface Sci. 1988, 122 (1), 209-219.

(8) Schebarchov, D.; Hendy, S. Capillary absorption of metal nanodroplets by single-wall carbon nanotubes. Nano Lett. 2008, 8 (8), 2253-2257.

(9) Schebarchov, D.; Hendy, S. Uptake and withdrawal of droplets from carbon nanotubes. Nanoscale 2011, 3 (1), 134-141.

(10) Yue, P.; Renardy, Y. Spontaneous penetration of a non-wetting drop into an exposed pore. Phys. Fluids 2013, 25 (5), 052104.

(11) Zhang, Z.; Chen, X.; Xu, J. Entry effects of droplet in a micro confinement: Implications for deformation-based circulating tumor cell microfiltration. Biomicrofluidics 2015, 9 (2), 024108.

(12) Benet, E.; Vernerey, F. J. Mechanics and stability of vesicles and droplets in confined spaces. Phys. Rev. E: Stat. Phys., Plasmas, Fluids, Relat. Interdiscip. Top. 2016, 94 (6), 062613.

(13) Zhang, Z.; Drapaca, C.; Chen, X.; Xu, J. Droplet squeezing through a narrow constriction: Minimum impulse and critical velocity. Phys. Fluids 2017, 29 (7), 072102.

(14) Amann-Hildenbrand, A.; Ghanizadeh, A.; Krooss, B. M. Transport properties of unconventional gas systems. Mar. Pet. Geol. 2012, 31 (1), 90-99.

(15) Singh, H. A critical review of water uptake by shales. J. Nat. Gas Sci. Eng. 2016, 34, 751-766.

(16) Striolo, A.; Cole, D. R. Understanding shale gas: Recent progress and remaining challenges. Energy Fuels 2017, 31 (10), 10300-10310.

(17) Huang, X.; Bandilla, K. W.; Celia, M. A. Multi-physics porenetwork modeling of two-phase shale matrix flows. Transp. Porous Media 2016, 111 (1), 123-141.

(18) Phan, A.; Cole, D. R.; Weiß, R. G.; Dzubiella, J.; Striolo, A. Confined water determines transport properties of guest molecules in narrow pores. ACS Nano 2016, 10 (8), 7646-7656.

(19) Zacharoudiou, I.; Chapman, E. M.; Boek, E. S.; Crawshaw, J. P. Pore-filling events in single junction micro-models with corresponding lattice Boltzmann simulations. J. Fluid Mech. 2017, 824, 550-573.

(20) Hu, J.; Ogletree, D.; Salmeron, M. The structure of molecularly thin films of water on mica in humid environments. Surf. Sci. 1995, 344 (3), 221-236.

(21) Hu, J.; Xiao, X.-D.; Ogletree, D.; Salmeron, M. Imaging the condensation and evaporation of molecularly thin films of water with nanometer resolution. Science 1995, 268 (5208), 267-269.

(22) Fang, C.; Zhang, F.; Qiao, R. Invasion of gas into mica nanopores: a molecular dynamics study. J. Phys.: Condens. Matter 2018, 30 (22), 224001. 
(23) Prakash, S.; Xi, E.; Patel, A. J. Spontaneous recovery of superhydrophobicity on nanotextured surfaces. Proc. Natl. Acad. Sci. U. S. A. 2016, 113 (20), 5508-5513.

(24) Berendsen, H.; Grigera, J.; Straatsma, T. The missing term in effective pair potentials. J. Phys. Chem. 1987, 91 (24), 6269-6271.

(25) Martin, M. G.; Siepmann, J. I. Transferable potentials for phase equilibria. 1. United-atom description of n-alkanes. J. Phys. Chem. B 1998, 102 (14), 2569-2577.

(26) Cygan, R. T.; Liang, J.-J.; Kalinichev, A. G. Molecular models of hydroxide, oxyhydroxide, and clay phases and the development of a general force field. J. Phys. Chem. B 2004, 108 (4), 1255-1266.

(27) Kroutil, O.; Chval, Z.; Skelton, A.; Predota, M. Computer simulations of quartz (101)-water interface over a range of $\mathrm{pH}$ values. J. Phys. Chem. C 2015, 119 (17), 9274-9286.

(28) Abraham, M. J.; Murtola, T.; Schulz, R.; Páll, S.; Smith, J. C.; Hess, B.; Lindahl, E. GROMACS: High performance molecular simulations through multi-level parallelism from laptops to supercomputers. SoftwareX 2015, 1, 19-25.

(29) Bussi, G.; Donadio, D.; Parrinello, M. Canonical sampling through velocity rescaling. J. Chem. Phys. 2007, 126 (1), 014101.

(30) Darden, T.; York, D.; Pedersen, L. Particle mesh Ewald: An N. $\log (\mathrm{N})$ method for Ewald sums in large systems. J. Chem. Phys. 1993, 98 (12), 10089-10092.

(31) Torrie, G. M.; Valleau, J. P. Nonphysical sampling distributions in Monte Carlo free-energy estimation: Umbrella sampling. J. Comput. Phys. 1977, 23 (2), 187-199.

(32) Kumar, S.; Rosenberg, J. M.; Bouzida, D.; Swendsen, R. H.; Kollman, P. A. The weighted histogram analysis method for freeenergy calculations on biomolecules. I. The method. J. Comput. Chem. 1992, 13 (8), 1011-1021.

(33) Allen, M. P.; Tildesley, D. J. Computer simulation of liquids; Oxford University Press: 2017.

(34) Firoozabadi, A.; Ramey, H. J., Jr Surface tension of waterhydrocarbon systems at reservoir conditions. J. Can. Pet. Technol. 1988, 27 (03), 41-48.

(35) Gravelle, S.; Ybert, C.; Bocquet, L.; Joly, L. Anomalous capillary filling and wettability reversal in nanochannels. Phys. Rev. E: Stat. Phys., Plasmas, Fluids, Relat. Interdiscip. Top. 2016, 93 (3), 033123.

(36) Israelachvili, J. N. Intermolecular and surface forces; Academic Press: 2011.

(37) Hu, H.; Weinberger, C. R.; Sun, Y. Effect of nanostructures on the meniscus shape and disjoining pressure of ultrathin liquid film. Nano Lett. 2014, 14 (12), 7131-7137.

(38) Hu, H.; Weinberger, C. R.; Sun, Y. Model of meniscus shape and disjoining pressure of thin liquid films on nanostructured surfaces with electrostatic interactions. J. Phys. Chem. C 2015, 119 (21), 11777-11785.

(39) König, P.-M.; Roth, R.; Mecke, K. Morphological thermodynamics of fluids: shape dependence of free energies. Phys. Rev. Lett. 2004, 93 (16), 160601.

(40) Jeong, J.-T.; Yoon, S.-H. Two-dimensional Stokes flow around a circular cylinder in a microchannel. J. Mech. Sci. Technol. 2014, 28 (2), 573-579.

(41) Gardiner, C. W. Handbook of Stochastic Methods, Vol 3; Springer: Berlin, 1985. 